\title{
DIFFUSION INDUCED INSTABILITY
}

\author{
BY \\ XANTHIPPI MARKENSCOFF \\ Department of Applied Mechanics Engineering Sciences, University of California, San Diego, \\ La Jolla, California
}

\begin{abstract}
It is shown that a coupled mechanochemical system can be stable under no diffusion and unstable with the addition of diffusion.
\end{abstract}

Introduction. The aim of this paper is to exhibit a curious instability phenomenon arising in a simple system of reaction-diffusion type. The curiosity lies in that, in the absence of diffusion, the system is stable, while the addition of diffusion, which commonly has a stabilizing effect, may induce instability.

The system describes conservation of linear momentum and energy in mixtures of two constituents, for instance, the equations governing the diffusion of hydrogen through an elastic matrix (Sofronis 1995, Li 1988). Later we shall also describe a very elementary mechanical model that exhibits similar behavior. For simplicity, we consider the case of a single space dimension, assuming that the body occupies the entire $x$-axis, $-\infty<$ $x<\infty$, and has (reference) density $\rho=1$. We shall be using throughout the following nomenclature. The displacement of the elastic matrix, denoted by $u$, induces strain $\varepsilon=\frac{\partial u}{\partial x}$ and velocity $v=\frac{\partial u}{\partial t}$. The stress will be denoted by $\sigma$, the concentration by $c$ and, its dual, chemical potential, by $\mu$. The state variables are $(\varepsilon, v, c)$, or, equivalently, $(\varepsilon, v, \mu)$. In the case where the state variables are $(\varepsilon, v, c)$, the constitutive relations are induced by the internal energy $\Phi=\Phi(\varepsilon, \sigma)$ through the thermodynamic relations

$$
\sigma=\left(\frac{\partial \Phi}{\partial \varepsilon}\right)_{c}, \quad \mu=\left(\frac{\partial \Phi}{\partial c}\right)_{\varepsilon} .
$$

In particular,

$$
\left(\frac{\partial \sigma}{\partial c}\right)_{\varepsilon}=\left(\frac{\partial \mu}{\partial \varepsilon}\right)_{c}
$$

On the other hand, when we use as state variables $(\varepsilon, v, \mu)$, the constitutive relations are induced by the Helmholtz free energy $\Psi=\Psi(\varepsilon, \mu), \Psi=\Phi-\mu c$, through

$$
\sigma=\left(\frac{\partial \Psi}{\partial \varepsilon}\right)_{\mu}, \quad c=-\left(\frac{\partial \Psi}{\partial \mu}\right)_{\varepsilon} .
$$

Received March 24, 1999.

2000 Mathematics Subject Classification. Primary 35B35, 35B40, 35Q72. 
In particular,

$$
\left(\frac{\partial \sigma}{\partial \mu}\right)_{\Sigma}=-\left(\frac{\partial c}{\partial \varepsilon}\right)_{\mu} .
$$

By the chain rule.

$$
\begin{aligned}
& \left(\frac{\partial \mu}{\partial c}\right)_{\xi}=\frac{1}{\left(\frac{i c_{c}}{\partial \mu}\right)_{\Sigma}}, \\
& \left(\frac{\partial \sigma}{\partial \mu}\right)_{\xi}=\left(\frac{\partial c}{\partial \mu}\right)_{\xi}\left(\frac{\partial \sigma}{\partial c}\right)_{\varepsilon} \\
& \left(\frac{\partial \sigma}{\partial \varepsilon}\right)_{\mu}=\left(\frac{\partial \sigma}{\partial \varepsilon}\right)_{c}-\frac{1}{\left(\frac{\partial \mu}{\partial c}\right)_{\xi}}\left(\frac{\partial \sigma}{\partial c}\right)_{\Sigma}^{2} .
\end{aligned}
$$

The governing conservation equations of linear momentum and energy for the medium, in Lagrangian coordinates, read

$$
\begin{aligned}
\frac{\partial^{2} u}{\partial t^{2}} & =\frac{\partial \sigma}{\partial x}, \\
\frac{\partial c}{\partial t} & =k \frac{\partial^{2} \mu}{\partial x^{2}},
\end{aligned}
$$

where $k \geq 0$ is a diffusion coefficient and where the time derivative denotes the total time derivative of a material volume. Upon substituting in (8) and (9) the constitutive function, we end up with a quasilinear system for the unknown state variables. Thus, if we use $(\varepsilon, v, c)$ as our state variables, we should consider the system

$$
\begin{aligned}
\frac{\partial^{2} u}{\partial t^{2}} & =\left(\frac{\partial \sigma}{\partial \varepsilon}\right)_{,} \frac{\partial^{2} u}{\partial x^{2}}+\left(\frac{\partial \sigma}{\partial c}\right)_{\Sigma} \frac{\partial c}{\partial x} \\
\frac{\partial c}{\partial t} & =k \frac{\partial}{\partial x}\left[\left(\frac{\partial u}{\partial \varepsilon}\right)_{,} \frac{\partial^{2} u}{\partial x^{2}}+\left(\frac{\partial u}{\partial c}\right)_{\Sigma} \frac{\partial c}{\partial x}\right]
\end{aligned}
$$

whereas in terms of the state variables $(\varepsilon, v, \mu)$ the system reads

$$
\begin{aligned}
\frac{\partial^{2} u}{\partial t^{2}} & =\left(\frac{\partial \sigma}{\partial \varepsilon}\right)_{\mu} \frac{\partial^{2} u}{\partial x^{2}}+\left(\frac{\partial \sigma}{\partial \mu}\right)_{\Xi} \frac{\partial \mu}{\partial x}, \\
k \frac{\partial^{2} \mu}{\partial x^{2}} & =\left(\frac{\partial c}{\partial \varepsilon}\right)_{\mu} \frac{\partial^{2} u}{\partial x \partial t}+\left(\frac{\partial c}{\partial \mu}\right)_{\Sigma} \frac{\partial \mu}{\partial t} .
\end{aligned}
$$

Needless to say, so long as the coefficients are interrelated by the proper relations (such as $(5) .(6)$. (7)). the above two systems (10)-(11) and (12)-(13) are equivalent, and whatever stability conclusions are derived for one apply automatically to the other. The aim here is to contrast stability properties in the absence of diffusion, i.e., $k=0$, with stability properties in the presence of diffusion, i.e., $k>0$.

Stability properties. In this (physically likely) situation where both elastic moduli $\left(\frac{\partial \sigma}{\partial \xi}\right)_{c}$ and $\left(\frac{\partial \sigma}{\partial \varepsilon}\right)_{\mu}$ are positive, we should expect stability. By contrast, in the (physically unlikely) situation in which both moduli $\left(\frac{\partial \sigma}{\partial \varepsilon}\right)_{c}$ and $\left(\frac{\partial \sigma}{\partial \varepsilon}\right)_{\mu}$ are negative, one should expect instability. But what happens if the modulus $\left(\frac{\partial \sigma}{\partial \xi}\right)_{\kappa}$ is positive while the modulus $\left(\frac{\partial \sigma}{\partial \varepsilon}\right)_{\mu}$ 
is negative? In that case, Eq. (10) would seem to predict stability, while Eq. (12) would seem to predict instability, and yet these two systems are equivalent. As we shall see. in the absence of diffusion, $k=0$, Eq. (10) prevails and dictates stability, while when diffusion is present, $k>0$, Eq. (12) prevails and dictates instability.

The case $k=0$. Let us first consider the case $k=0$. It is simpler to examine stability in the context of the system (10)-(11). Indeed, Eq. (11) immediately yields $c(x, t)=c(x, 0)$, in which case Eq. (10) reduces to the standard wave equation in the single unknown variable $u$ with given forcing term. We thus have stability if and only if the elasticity modulus $\left(\frac{\partial \sigma}{\partial \varepsilon}\right)_{c}$ is positive. As noted above, under the assumption $\left(\frac{\partial \sigma}{\partial \xi}\right)_{c}>0$ the same conclusion of stability should hold automatically for the equivalent system (12)(13). It will be instructive, however, to rederive this result directly. Toward that end, we rewrite (12)-(13) (with $k=0$ ) in the equivalent linearized (about a constant state) canonical form

$$
\partial_{\iota}\left(\begin{array}{l}
\varepsilon \\
v \\
\mu
\end{array}\right)+\left(\begin{array}{ccc}
0 & -1 & 0 \\
-\alpha & 0 & -\beta \\
0 & \frac{3}{\gamma} & 0
\end{array}\right) \partial_{x}\left(\begin{array}{l}
\varepsilon \\
v \\
\mu
\end{array}\right)=0
$$

where we have set

$$
\alpha=\left(\frac{\partial \sigma}{\partial \varepsilon}\right)_{\mu}, \quad \beta=\left(\frac{\partial \sigma}{\partial \mu}\right)_{\varepsilon}=-\left(\frac{\partial c}{\partial \varepsilon}\right)_{\mu}, \quad \gamma=\left(\frac{\partial c}{\partial \mu}\right)_{\Sigma},
$$

all evaluated at the constant state $(\bar{\varepsilon}, \bar{\mu})$. Stability will prevail if and only if the system (14) is hyperbolic, that is, the eigenvalues of the matrix

$$
\left(\begin{array}{ccc}
0 & -1 & 0 \\
-\alpha & 0 & -\beta \\
0 & \frac{-\beta}{\gamma} & 0
\end{array}\right)
$$

(characteristic speeds), are all real. A simple calculation yields that the three characteristic speeds are $\lambda_{1}=0$ and $\lambda_{2.3}= \pm\left(\alpha+\frac{\beta^{2}}{\gamma}\right)^{1 / 2}$. Therefore, we have stability if and only if

$$
\left(\frac{\partial \sigma}{\partial \varepsilon}\right)_{\mu}+\frac{1}{\left(\frac{\partial c}{\partial \mu}\right)_{\varepsilon}}\left(\frac{\partial \sigma}{\partial \mu}\right)_{\varepsilon}^{2}>0
$$

By virtue of $(5),(6)$, and $(7),(16)$ is equivalent to $\left(\frac{\partial \sigma}{\partial \xi}\right)_{c}>0$, as was to be expected.

The case $k>0$. What is remarkable, contrary to intuition, is that we may have stability, in the absence of diffusion, even when the elastic modulus $\left(\frac{\partial \sigma}{\partial \varepsilon}\right)_{\mu}$ may be negative, provided of course that the other derivatives compensate so that (16) still holds. We now proceed to consider the case $k>0$. As we shall see, in that case we have instability, i.e., when $\left(\frac{\partial \sigma}{\partial \varepsilon}\right)_{\mu}<0$ even though (16) may hold. We rewrite the system (12)-(13) using the notation (15), namely

$$
\begin{gathered}
\frac{\partial^{2} u}{\partial t^{2}}=\alpha \frac{\partial^{2} u}{\partial x^{2}}+\beta \frac{\partial \mu}{\partial x} \\
\gamma \frac{\partial \mu}{\partial t}-\beta \frac{\partial^{2} u}{\partial x \partial t}=k \frac{\partial^{2} \mu}{\partial x^{2}}
\end{gathered}
$$


and seek particular solutions that are oscillatory in space while exponentially fast in time, namely,

$$
u(x, t)=a e^{i \xi x} e^{n t}, \quad \mu(x, t)=b e^{i \xi x} e^{n t},
$$

where $\xi$ is a real parameter and $n$ is a large positive integer. Substituting into (17) yields

$$
\begin{aligned}
\left(n^{2}+\alpha \xi^{2}\right) a-i \beta \xi b & =0, \\
-i \beta n \xi a+\left(\gamma n+k \xi^{2}\right) b & =0,
\end{aligned}
$$

whence we obtain the characteristic equation

$$
\alpha k \xi^{4}+n\left(k n+\alpha \gamma+\beta^{2}\right) \xi^{2}+\gamma n^{3}=0 .
$$

The nature of the roots of (20) will depend on the signs of $\alpha$ and $\gamma$, namely (recall (15)) the sign of the moduli $\left(\frac{\partial \sigma}{\partial \varepsilon}\right)_{\mu}$ and $\left(\frac{\partial c}{\partial \mu}\right)_{\varepsilon}$. When $(20)$ possesses real roots for arbitrarily large $n$, we shall establish Hadamard-type instability. On the other hand, when all roots $\xi$ of (20), for large $n$, are complex, no such instability should be expected. Clearly, the roots $\xi$ of (20) when both $\alpha$ and $\gamma$ are positive are complex. Also notice that when $\alpha<0$, then, by virtue of (7), $\gamma$ is necessarily positive, so long as we want to preserve the modulus $\left(\frac{\partial \sigma}{\partial \varepsilon}\right)_{c}>0$. Therefore, the only possibilities for instabilities arise when $\alpha>0$, $\gamma<0$, or $\alpha<0, \gamma>0$. When $\alpha>0, \gamma<0$, (20) admits roots with

$$
\xi_{n}^{2}=-\frac{\gamma}{k} n+O(1)
$$

while, when $\alpha<0, \gamma>0,(20)$ admits roots with

$$
\xi_{n}^{2}=-\frac{1}{\alpha} n^{2}+\frac{\beta^{2}}{\alpha k} n+O(1) .
$$

Let us establish Hadamard instability in the second case, the first one being, of course, mathematically similar. We start with the initial data

$$
u(x, 0)=\sum_{n=1}^{\infty} a_{n} e^{i \xi_{n} x}, \quad \mu(x, 0)=\sum_{n=1}^{\infty} b_{n} e^{i \xi_{n} x}
$$

where

$$
a_{n}=\frac{1}{n^{4}}, \quad b_{n}=\frac{1}{i \beta \xi_{n}}\left(n^{2}+a \xi_{n}^{2}\right) a_{n} \cong-i \frac{\sqrt{-\alpha} \beta}{k} \frac{1}{n^{4}}
$$

so that $u(x, 0)$ and $\mu(x, 0)$ are twice continuously differentiable. It is clear that for such initial data the solution blows up to infinity instantaneously.

A different perspective of the destabilizing effect of diffusion is provided by the following argument. The energy equation, derived by combining Eqs. (8) and (9) and using the constitutive relations (1), is

$$
\frac{\partial}{\partial t}\left[\frac{1}{2} v^{2}+\Phi(\varepsilon, c)\right]=\frac{\partial}{\partial x}(v \sigma)+\frac{k}{2} \frac{\partial^{2} \mu}{\partial x^{2}}-k\left(\frac{\partial \mu}{\partial x}\right)^{2} .
$$


Upon integrating the above equation over space and time, assuming that $\mu$ is constant at infinity, we obtain

$$
\begin{aligned}
\int_{-\infty}^{\infty} & {\left[\frac{1}{2} v^{2}(x, t)+\Phi(\varepsilon(x, t), c(x, t))\right] d x+k \int_{0}^{t} \int_{-\infty}^{\infty}\left(\frac{\partial \mu}{\partial x}\right)^{2} d x d t } \\
= & \int_{-\infty}^{\infty}\left[\frac{1}{2} v^{2}(x, 0)+\Phi(\varepsilon(x, 0), c(x, 0))\right] d x .
\end{aligned}
$$

Assuming that the internal energy $\Phi$ is nonnegative, identity (26) implies that $\frac{\partial \mu}{\partial x}(x)$ will become very small for large $t$. Then, in that case, referring to Eq. (12), we infer that the second term in the right-hand side of (12) becomes eventually negligible, and so, whenever the modulus $\left(\frac{\partial \sigma}{\partial \varepsilon}\right)_{\mu}$ is negative, instability will occur.

Conclusion. What we showed above is surprising. Indeed, it is commonly assumed (for good reason) that diffusion has a stabilizing effect. Here the opposite occurs. This is of course a consequence of our assumption, that even though $\left(\frac{\partial \sigma}{\partial \varepsilon}\right)_{c}>0$, we have $\left(\frac{\partial \sigma}{\partial \varepsilon}\right)_{\mu}<$ 0 . The natural question arises whether constitutive relations with such properties may occur in physical models governed by such equations. The answer seems not to be known in the context of the model of diffusion of hydrogen through an elastic matrix (which motivated our discussions in the first place), due to the difficulty of determining experimentally the elastic modulus $\left(\frac{\partial \sigma}{\partial \varepsilon}\right)_{\mu}$. However, we describe below a simple, though somewhat artificial, purely mechanical model, in which $\left(\frac{\partial \sigma}{\partial \varepsilon}\right)_{\mu}<0$ may occur. This model is due to J. R. Rice (private communication). We assume that our medium is a mixture of an elastic matrix with cracks filled with fluid under pressure. In that case, the role of the "chemical potential" is played by the pressure of the fluid in the cracks. To estimate the modulus $\left(\frac{\partial \sigma}{\partial \varepsilon}\right)_{\mu}<0$, we imagine that the medium is immersed in an infinite reservoir containing fluid at a constant pressure $\mu$. Stretching of the specimen will induce opening of the cracks, the pressure of the fluid remaining constant, and thus $\sigma$ will decrease, i.e., $\left(\frac{\partial \sigma}{\partial \varepsilon}\right)_{\mu}<0$.

Acknowledgment. The author is grateful to Professor J. R. Rice of Harvard University for helpful discussions and for the hospitality during her sabbatical at Harvard.

\section{REFERENCES}

[1] P. Sofronis, The influence of mobility of dissolved hydrogen on the elastic response of a metal, Journal of Mechanics and Physics of Solids 43, 1385-1407 (1995)

[2] J. M. C. Li, Negative creep and mechanochemical spinodal in amorphous metals, Journal of Materials Science and Engineering 98, 465-468 (1988) 